\title{
DOS NUEVOS TEXTOS IBÉRICOS DEL SUR DE FRANCIA
}

Jürgen Untermann

\section{LA PIEDRA DE CRUZY / ENSÉRUNe}

Con la "piedra de Cruzy" se presenta un monumento único en la epigrafía ibérica, tanto por su forma exterior como por su texto, hallado en 1963 en un lugar desconocido muy cerca del oppidum de Ensérune. Permaneció unos años en una casa particular de la región, y hace poco fue entregado al Museo Municipal de Cruzy, una pequeña ciudad del Département de l'Hérault, $15 \mathrm{~km}$ al oeste del lugar del hallazgo. Debo su conocimiento a M. Jean Valdeyron, de Cruzy, quien me facilitó la autopsia de la pieza el día 17 de marzo de 1998.

Se trata de un gran bloque de piedra aproximadamente rectangular $(40 \times 25 \mathrm{~cm})$, demasiado grueso $(35 \mathrm{~cm})$ para ser una estela sepulcral, y tampoco presenta las características propias de un altar o del pedestal de una estatua. Además, los trazos de las letras carecen totalmente de rasgos monumentales, y recuerdan más bien los grafitos en los que los turistas actuales dejan sus huellas sobre las paredes de monumentos históricos. Podría imaginarse que estemos frente a un sillar pertenciente a la fortificación del oppidum de Ensérune, en el que alguién grabó unas treinta letras en tres líneas, usando cincel y martillo, aunque sin muchas pretensiones estéticas o caligráficas.

El dibujo se basa en un dibujo de Jean Valdeyron, ligeramente modificado según la autopsia. ${ }^{2}$ La transcripción resulta ser la siguiente:

\section{(bo?)iubebaŕete \\ neitiniunstir : kule \\ śare : $\mathbf{a}<\mathbf{r}>$ kiteibase}

\footnotetext{
${ }^{1}$ Además, M. J. Valdeyron tuvo la amabilidad de incluir un breve comentario lingüístico y epigráfico, que le había puesto a disposición, en su publicación provisional de la piedra (Valdeyron 1998). Un tratamiento más detenido está en prensa en la revista Archéologie en Languedoc.

${ }_{2}^{2}$ J.Valdeyron reconoció seis letras al final de las líneas 1 y 2 , que a mi modo de ver corresponden a imperfecciones casuales de la superficie.
} 


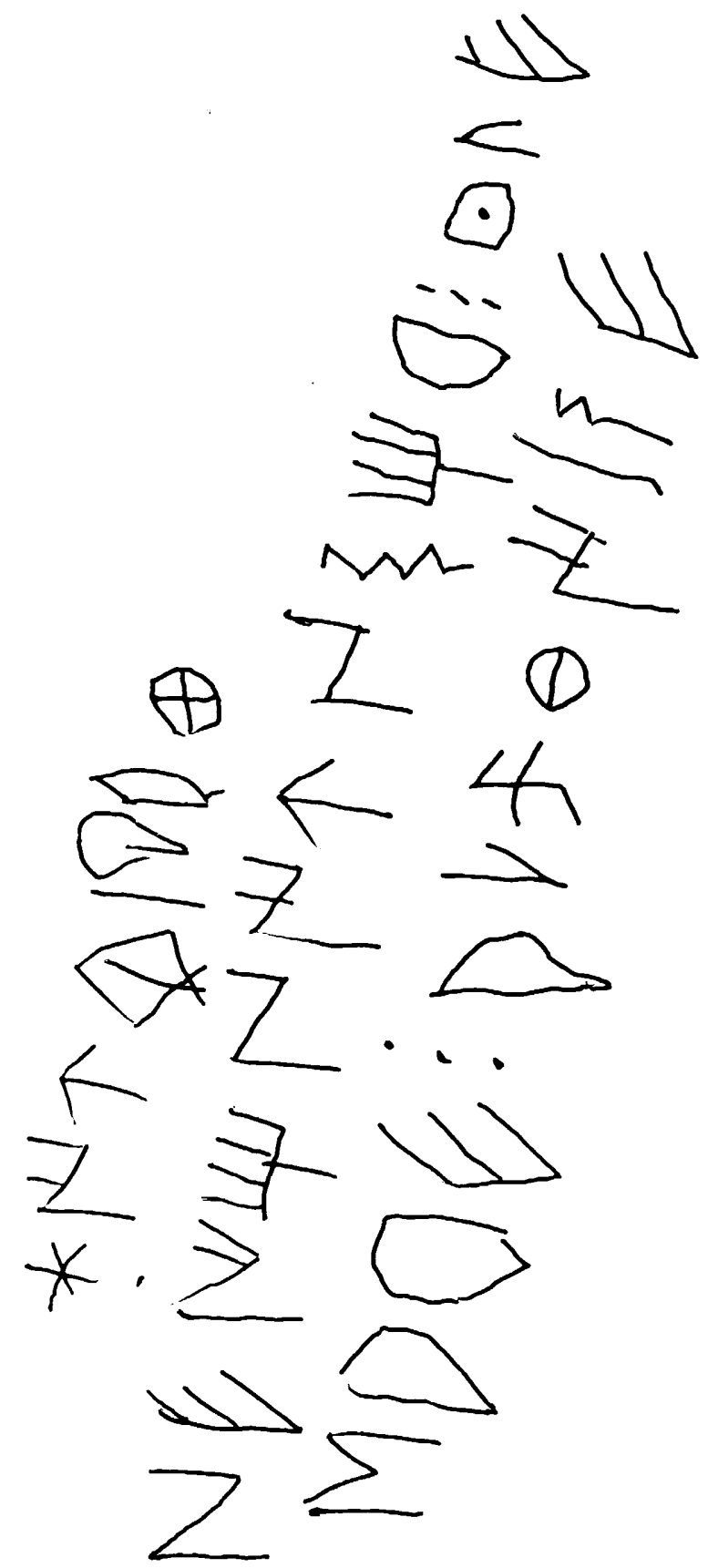


Al inicio del primer renglón se ven tres trazos muy delgados formando una estrella que podría ser la letra ibérica bo, pero muestran un relieve mucho menos profundo que los demás signos, así que prefiero suponer que no tienen que ver nada con el texto. Las tres últimas letras son muy mal legibles, pero no dan lugar a otras posibilidades más probables de identificación. En el tercer renglón la sexta letra parece incompleta: me parece, que el grabador, al empezar a repetir la letra precendente (a), se dió cuenta de su error y la dejó sin acabar en vez de sustituirla por la forma correcta; del contexto se desprende que debe tratarse de una $\mathbf{r}$.

Respecto a la primera línea sólo puedo ofrecer una hipótesis extremadamente atrevida: dado que -te es un sufijo que se une frecuentemente con antropónimos, ${ }^{3}$ me parece lícito intentar buscar en la secuencia iubebaŕe un nombre de persona. Hay que acordarse del hecho de que en la epigrafía ibérica de Ensérune el fonema $/ \mathrm{m} /$ de nombres galos puede ser expresado mediante los signos silábicos con $\mathbf{b},{ }^{4}$ p.e. kobakie - Comagios (MLH B.1.53), latubaŕe - Latumaros (MLH B.1.364), katubaŕe - Catumaros sobre el plomo de Ensérune. ${ }^{5}$ Por lo tanto, aquí también, -baŕe puede corresponder a la palabra gala māros; en cambio, es más difícil el caso de iube: tal vez pueda contarse con un adjetivo galo *iūmo- < *iuvamo- (= antiguo irlandés óam, galés ieuaf), superlativo del adjetivo *ieu- "joven".

Al final del texto sigue la denominación muy clara de una persona mediante dos nombres, el suyo y el de su padre, kuleśar-e arkiteibas-e, que llevan los dos el sufijo -e, igualmente bien atestiguado junto con antropónimos, ${ }^{6}$ pero todavía de función desconocida. Se trata de nombres compuestos ibéricos, que contienen los componentes bien conocidos $^{7}$ kuleś, śar, arki y teibas, variante ortográfica de tibas.

La novedad más llamativa, que debemos a la piedra de Cruzy, es el nuevo testimonio de la secuencia neitiniunstir. Ya la conocemos desde hace mucho tiempo por el rhyton de Ullastret (MLH C.2.8), y recientemente apareció dos veces en el plomo Marsal: ${ }^{9}$ se da al inicio de las dos versiones de la cara A, en el texto inferior escrita como aquí y en Ullastret, en el texto superior separada por puntos, neitin : iunstir. ${ }^{10}$ Pero hay que confesar que, a pesar de este número considerable de testimonios,

\footnotetext{
${ }^{3}$ Posiblemente indicando una función ergativa: aparece varias veces delante la palabra ekiar que significa "ha fabricado" o "es la obra de": MLH III. 1, 186.

${ }^{4}$ Quintanilla 1998, 195.

${ }^{5}$ Publicado por Solier-Barbouteau 1988, 73-85.

${ }^{6}$ MLH III. 1,163 y ss.

${ }^{7}$ Cp. MLH III.1, 227 (kuleś), 211 (arki), 234sg. (tibas); śar está atestiguado por iltiŕ-śar sobre un plomo de Pech-Maho (Solier 1979, 82) aŕbi-śar sobre un pondus de Oliete (Silgo 2001, 348), tolo-śar en las listas del Bronce Botorrita III (Untermann 1996, 159).

${ }^{8}$ Véase Quintanilla 1998, 140-142.

${ }^{9}$ Publicado y comentado por Untermann 1998.

${ }^{10}$ Además, no es seguro pero tampoco imposible, que al inicio del gran plomo de Ampurias (Sanmartí 1988) haya que completar nei]tin : iunstir.
} 
siguen siendo oscuros tanto el significado como la función contextual de neitiniunstir. ${ }^{11}$

\section{El Plomo de MonTlaurès}

Hace unos años, en el sitio ibérico de Montlaurès, pocos kilómetros al norte de Narbonne, ${ }^{12}$ salió a la luz una pequeña banda de plomo enrollada, que debe ser la extremidad de una banda mucho más larga. La parte conservada desenrollada mide entre 26 y $30 \mathrm{~mm}$ de ancho y $78 \mathrm{~mm}$ de largo. Deja ver letras ibéricas a los dos lados, bastante bien legibles en una cara (A), casi totalmente borradas en la otra (B). El 9 de Noviembre de1998 tuve la oportunidad de estudiarla detenidamente gracias a la amabilidad de M. Michel Feugère, director del Centro Arqueológico del CNRS en Montagnac (Hérault). Actualmente se conserva en una colección particular de Nîmes.

El texto de la cara A está dividido en dos secciones mediante una línea que atraviesa la lámina en dirección casi vertical, a una distancia de 50 a $46 \mathrm{~mm}$ del margen correctamente recortado a la derecha. A la izquierda de esta línea se ven los últimos signos del texto principal: una letra del primer renglón, tres del segundo, y dos del tercero. En la sección de la derecha se encuentran tres palabras completas, igualmente en tres renglones, que evidentemente contienen una nota final añadida al mensaje del texto precedente.

Las formas de las letras coinciden casi perfectamente con las del gran plomo de Ullastret (MLH C.2.3), nótense en particular los signos de ŕ, s, be y ka (pertenecen, pués, a la fase "preclásica" de la epigrafía ibérica). Resulta inseguro el valor de la penúltima letra del segundo renglón: por una parte puede ser una variante arcaica de o, que aparece también en la inscripción arcaizante del famoso vaso de Liria MLH F.13.13, ${ }^{13}$ y en uno de los plomos de Los Villares (MLH F.17.1), por otra parte recuerda el signo que representa bu en dos de los plomos de Pech-Maho, que sólo se distingue por mostrar un punto en el centro de cada uno de los cuadrados que componen el signo. ${ }^{14}$

La transcripción de la cara A no ayuda a despejar la duda:

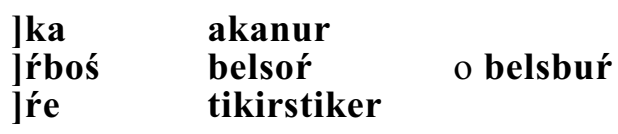

Sobre -ka y -ŕe sólo se puede decir, que son segmentos bien atestiguados que funcionan como sufijos al final de palabras ibéricas. ${ }^{15}$

\footnotetext{
${ }^{11}$ Para una discusión más detallada de los contextos véase Untermann 2001, 617-621.

${ }^{12}$ Para la descripción del sitio y para los hallazgos epigráficos anteriores véase MLH II pp.311-317.

${ }^{13}$ kutur : oisor, leído por P.Beltrán gudua deisdea: véase MLH III.2, 463.

${ }^{14}$ Solier 1979, 71.

${ }^{15}$ Muy frecuente -ka (MLH III.1,166-168); con 8 testimonios más o menos seguros -ŕe (MLH III.1, 174).
} 
Dos nuevos textos ibéricos del Sur de Francia

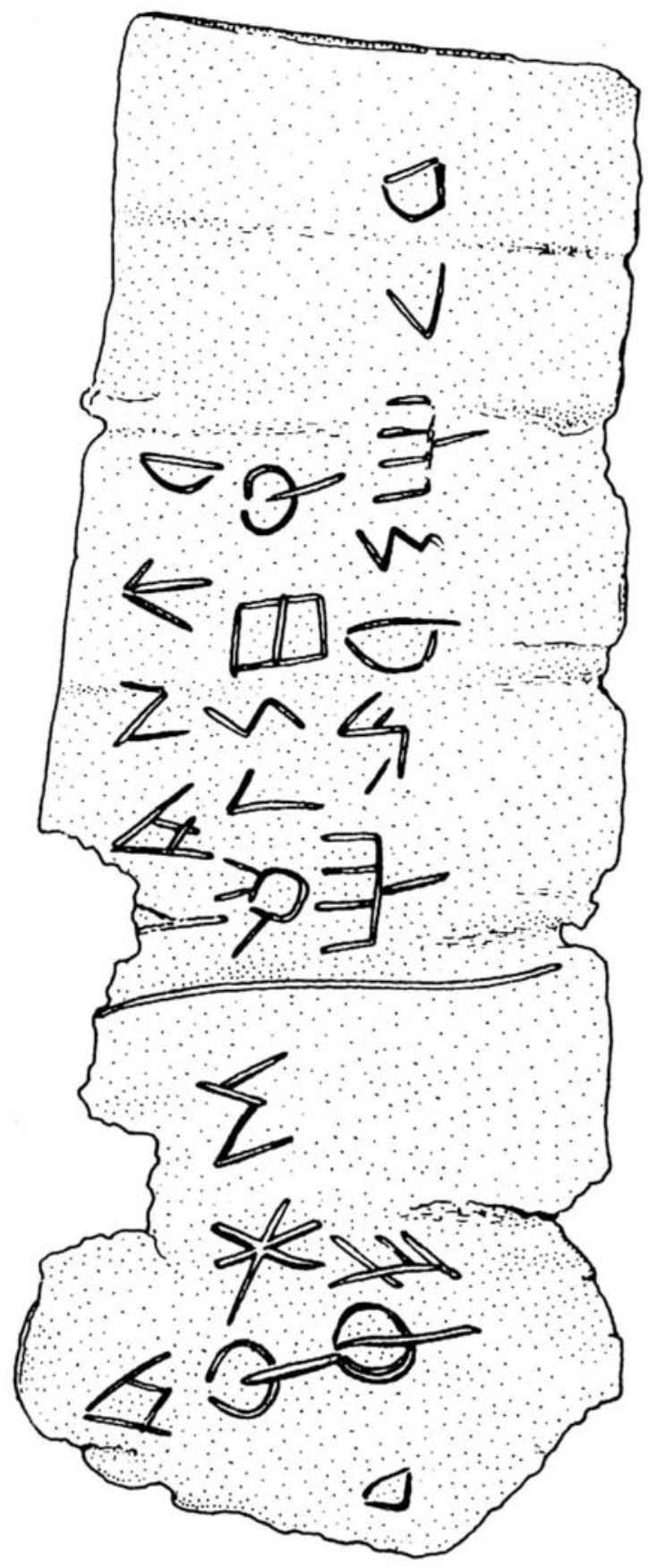

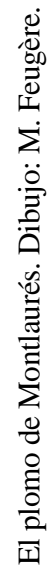




\section{Jürgen Untermann}

En la sección de la derecha se presenta la persona, que evidentemente es la responsable del texto del plomo: belsoŕ (o belsbuŕ), hijo de tikirstiker. ${ }^{16}$ Las dos palabras pueden ser analizadas como antropónimos ibéricos compuestos: para tikirs recuérdese tikirs-balaur en Palamós (MLH C.4.2) y tikirs-(s)eni en Sagunto (MLH F.11.10), tiker vuelve a aparecer en aŕki-tiker en Castellón (MLH F.6.1) y iltiŕ-tiker sobre un plato de Abengibre (MLH G.16.1). El primer componente de belsoŕ/belsbuŕ se encuentra en an-bels de Ensérune (MLH B.1.40) o en el nombre de un caballero de la Turma Salluitana, Adimels < adinbels. ${ }^{17}$ En el caso de que belsoŕ sea la transcripción correcta, el compuesto se analiza como bels-soŕ y recuerda los antropónimos soŕ-ike sobre la estela de Vic (MLH D.2.1) y kani-soŕ sobre uno de los plomos de Pech-Maho; ${ }^{18}$ por otro lado, un componente antroponímico buŕ está atestiguada por atin-buŕ y kuleś-bur sobre plomos de Pech-Maho ${ }^{19}$ y por el nombre "breve" ("Kurzname") ${ }^{20}$ Bur-do de la Turma Salluitana.

En fin, akanur no muestra ninguna relación con el repertorio de los nombres de personas ibéricos. En cambio, la posición en la frase final del texto y la terminación -ur son rasgos en común con la palabra kutur que se da cinco veces en textos ibéricos sobre plomo, ${ }^{21}$ tres de ellas en los sintagmas con los que terminan los respectivos textos:

última línea del plomo V de Pech-Maho: ${ }^{22}$

\section{jitiatu : ban : kutur : biteŕokan}

las dos últimas líneas del plomo de Gruissan: ${ }^{23}$

$$
\text { ...: biteian : keitiŕeie[.....]/ kutuŕaŕ[... }
$$

la parte final del nuevo plomo de La Bastida de Moixent: ${ }^{24}$

\section{-biteŕtuan koikakiskutuŕ.}

Sé muy bien que estas citas no tienen ningún valor probatorio directo para la interpretación de akanur: las aduzco a título de ejemplo de un formulario empleado al final de textos sobre plomo, cuyo elemento constante es una palabra, que termina en -ur, quizá de una categoría grama-

\footnotetext{
${ }^{16}$ Conforme a la fórmula normal de la antroponimia ibérica: MLH III.1, 197.

${ }^{17}$ Sobre $m=n+b$ véase en último lugar Quintanilla 1998, 196.

${ }^{18}$ Solier 1979,83 , con transcripción errónea kenisoŕ.

${ }^{19}$ Solier 1979, 80, 83 .

${ }^{20}$ Sobre este tipo de antropónimos véase MLH III. 1, $203 \mathrm{sg}$.

${ }^{21}$ Además una vez en la inscripción pintada F.13.13 del vaso de Liria ya mencionado en la nota 13.

${ }^{22}$ Solier-Barbouteau 1988, 71 sgs.

${ }^{23}$ Solier-Barbouteau 1988, 91.

${ }^{24}$ La publicación por D. Fletcher y H. Bonet todavía está en prensa; véase la nota provisonal de Faria 1992/93, 278.
} 
tical, que corresponde a la del verbo en las lenguas indoeuropeas. Tal vez akanur tenga una función que no sea muy distinta de aquella que kutur parece tener en los textos que acabo de enumerar.

\section{BIBLIOGRAFÍA}

Faria 1992/93 = MARQUES DE FARIA, A., "Notas a algumas inscrições ibéricas recentemente publicadas." Portugalia 13/14, pp. 277-279.

MLH = Monumenta Linguarum Hispanicarum. Herausgegeben von J. Untermann. Band I-IV, Wiesbaden 1975-1997.

Quintanilla 1998 = QuINTANILLA, A., Estudios de fonología ibérica, Anejos de VELEIA. Series minor 11. Vitoria.

Sanmartí 1988 = SANMARTÍ-GREGO, E., "Una carta en lengua ibérica, escrita sobre plomo, procedente de Emporion." RANarb 21, pp. 95-113.

Silgo 2001 = SILGO GAUCHE, L., "Grafitos ibéricos de El Palomar (Oliete, Teruel)." Palaeohispanica 1, pp. 347-352.

Solier 1979 = SOLIER, Y., "Découverte d'inscriptions sur plombs en écriture ibérique dans un entrepôt de Pech-Maho (Sigean)." RANarb 12, pp. 55-123.

Solier-Barbouteau 1988 = Solier, Y., Barbouteau, H. "Découverte de nouveaux plombs, inscrits en ibère, dans la région de Narbonne." RANarb 21, pp. 61-94.

Untermann $1996=$ UNTERMANN, J., "Onomástica." En: Beltrán,F., de Hoz, J., Untermann, J. El tercer bronce de Botorrita (Contrebia Belaisca), Zaragoza, pp. 109-166.

Untermann 1998 = UNTERMANN, J., "Comentario sobre una lámina de plomo con inscripción ibérica de la colección D.Ricardo Marsal, Madrid." Habis 19, pp. 7-21.

Untermann 2001 = UNTERMANN, J., "Algunas novedades sobre la lengua de los plomos ibéricos." En: Religión, lengua y cultura prerromanas de Hispania. Actas del VIII Coloquio sobre lenguas y culturas paleohispánicas (Salamanca 1999), Salamanca, pp. 613-628.

Valdeyron 1998 = VALDEYRON, J., "Pierre ibérique du Musée de Cruzy (F.34)." Association culturelle, archéologique et paléontologique de Cruzy (sin fecha y número, 1998), 4 páginas. 